XIth International Symposium on Luminescende Spectrometry in Biomedical and Environmental Analysis Spectrosopic and Imaging Detection Techniques, Tsinghua University, Beijing, China. Information: Professor Dr. Xinrong Zhang, Analysis Center, Department of Chemistry, Tsinghua University, Beijing 100084, P. R. China. Tel. +86 (10) 62781688 (Lab); Fax+86 (10)62770327 (Lab). E-Mail: xrzhang@chem.tsinghua.edu.cn $\mathbf{2 8}^{\text {th }}$ International Symposium \& Exhibit on High Performance Liquid Phase Separations \& Related Techniques, Philadelphia Convention Center, Philadelphia, Pennsylvania, USA. Information: janetbarr@aol.com

\title{
Erratum
}

\section{Determination of Clenbuterol in Horse Hair by Gas Chromatography - Tandem Mass Spectrometry}

by M. A. Popot / S. Boyer / P. Maciejewski / P. Garcia / Y. Bonnaire / L. Beyer / D. Lesage / J. C. Tabet

published in 2001, 53, S-375-S-378

The sentence on page S-377, column 1, line 12 should read:

"This competition has been demonstrated by using MAB (metastable atom bombardement) ionization techniques [12] which enables production of the odd-electron molecular ion $\left(\mathrm{M}^{+}\right)$." 